\title{
An oral formulation of cilostazol nanoparticles enhances intestinal drug absorption in rats
}

\author{
CHIAKI YOSHIOKA, YOSHIMASA ITO and NORIAKI NAGAI
}

Faculty of Pharmacy, Kindai University, Higashi-Osaka, Osaka 577-8502, Japan

Received August 7, 2017; Accepted October 16, 2017

DOI: $10.3892 / \mathrm{etm} .2017 .5373$

\begin{abstract}
Cilostazol (CLZ) is an anti-platelet agent that is generally used after the onset of cerebral infarction. However, CLZ is a poorly water-soluble drug and a strategy for increasing its bioavailability is required. In the present study, novel oral formulations were designed containing CLZ solid nanoparticles to improve bioavailability. The present study investigated the therapeutic effect of the oral formulations containing CLZ nanoparticles on ischemic stroke using a cerebral ischemia/reperfusion-induced injury model (MCAO/reperfusion mice). The oral formulation containing CLZ nanoparticles (CLZ/ $\mathrm{R}_{\text {nano }}$ tablet) was prepared using a combination of recrystallization and ball milling with the following ingredients: CLZ, docusate sodium, methylcellulose, 2-hydoxypropyl- $\beta$-cyclodextrin, gum arabic, polyvinylpyrrolidone, and mannitol. The particle size after re-dispersion of the $C L Z / R_{\text {nano }}$ tablet was $64 \pm 47 \mathrm{~nm}$ (mean \pm standard deviation). The CLZ areas under the concentration-time curve (AUC) and mean residence time (MRT) in rats that were administered $\mathrm{CLZ} / \mathrm{R}_{\text {nano }}$ tablets were significantly greater compared with those in rats that were administered $\mathrm{CLZ} / \mathrm{R}_{\text {micro }}$ tablets. Results indicated, the AUC after administration of CLZ/R $\mathrm{R}_{\text {nano }}$ tablets was 3.1-fold higher compared with that after administration of the commercially available CLZ OD tablet. In addition, oral administration with $C L Z / R_{\text {nano }}$
\end{abstract}

Correspondence to: Dr Noriaki Nagai, Faculty of Pharmacy, Kindai University, 3-4-1 Kowakae, Higashi-Osaka, Osaka 577-8502, Japan

E-mail: nagai_n@phar.kindai.ac.jp

Abbreviations: AUC, concentration-time curve; AUMC, area under the first moment curve; CLZ, cilostazol; CLZ/R, recrystallized cilostazol; DS, docusate sodium; HP $\beta C D$, 2-hydoxypropyl$\beta$-cyclodextrin; $\mathrm{k}_{\mathrm{a}}$, absorption rate constant; $\mathrm{MC}$, methylcellulose; $\mathrm{MCAO} /$ reperfusion, middle cerebral arterial occlusion followed by reperfusion; MRT, mean residence time; PLGA, poly (lacticcoglycolic acid); PVP, polyvinylpyrrolidone; $\mathrm{RNP}^{\mathrm{O}}$, redox nanoparticles; SEM, scanning electron microscope; TTC, 2,3.5-triphenyl tetrazolium chloride; XRD, powder X-ray diffraction

Key words: cilostazol, nanoparticle, oral formulation, ischemic stroke, polymorphic form tablets ameliorated neurological deficits caused by ischemic stroke in MCAO/reperfusion mice. It is possible that the oral formulation containing CLZ nanoparticles will be useful for the treatment of patients with ischemic stroke and that these findings will provide significant information that can be used to improve the drug with low bioavailability.

\section{Introduction}

Cilostazol (CLZ) has anti-platelet aggregation and vasodilatory effects with minimal cardiac effects (1). In clinical studies, CLZ has been used as a therapeutic agent for improving symptoms in conditions such as cancer (2) and pain accompanied by chronic arterial obstruction (3) and for ameliorating (4) and preventing cerebral infarction (5). In addition, recent studies indicated that CLZ reduced the degree of neuronal cell death after transient cerebral ischemia (6). However, CLZ is poorly water-soluble (the solubility is $4.83 \mu \mathrm{g} / \mathrm{ml}$ in the water at $37^{\circ} \mathrm{C}$ ) and its bioavailability is low (7). Therefore, the development of a strategy for increasing the bioavailability of CLZ is needed.

Recently, many methods using poly (lactic-coglycolic acid) (PLGA) nanospheres $(8,9)$, redox nanoparticles $\left(\mathrm{RNP}^{\mathrm{O}}\right)(10)$, emulsions (11), and polymer micelles (12) have been evaluated for improving the bioavailability of poorly water-soluble drugs. We also reported a method for producing drug nanoparticles using the ball and bead mill (13-18) and showed that indomethacin solid nanoparticles exhibited enhanced drug bioavailability after oral administration (17). We hypothesized that an oral formulation of CLZ nanoparticles would provide high drug absorption and that enhancing the bioavailability of CLZ would increase its effectiveness for preventing neuronal cell death after transient cerebral ischemia. In addition, we expected this formulation would lead to a decrease in the required amount of orally administered CLZ.

It is important to carefully select a method for preparing the drug nanoparticles. Conventional milling methods, such as planetary ball milling, have been widely used in the drug nanoparticle processing field $(19,20)$. In addition, a combination of recrystallization and ball milling can be used to prepare large amounts of fine drug nanoparticles at a low price (21). In this study, we designed new oral formulations containing CLZ solid nanoparticles using a combination of recrystallization and ball mill methods. Moreover, we investigated the usefulness of these formulations by evaluating drug bioavailability 
and their protective effect in ischemic stroke using a cerebral ischemia/reperfusion-induced injury model (MCAO/reperfusion mice).

\section{Materials and methods}

Materials. CLZ was kindly provided by Otsuka Pharmaceutical Co., Ltd. (Tokyo, Japan). 2-Hydoxypropyl- $\beta$-cyclodextrin $(\mathrm{HP} \beta \mathrm{CD})$ was purchased from Nihon Shokuhin Kako Co., Ltd. (Tokyo, Japan). Low-substituted methylcellulose (MC) was provided by Shin-Etsu Chemical Co., Ltd. (Tokyo, Japan). Docusate sodium (DS) was obtained from Sigma Co., Inc. (Tokyo, Japan). All other chemicals used were of the highest purity commercially available.

Animals. Wistar rats (7 weeks, male) and ICR mice (6 weeks, male) were housed under standard conditions (fluorescent light 07:00 a.m.-07:00 p.m., $25 \pm 1^{\circ} \mathrm{C}$ ) and allowed free access to a commercial diet (CR-3; Clea Japan Inc., Tokyo, Japan) and water. All procedures were performed in accordance with the Kindai University School of Pharmacy Committee for the Care and Use of Laboratory Animals, and the study received ethical approval form the Kindai University School of Pharmacy Ethics Committee (Osaka, Japan). In this study, we administered $3 \mathrm{mg} / \mathrm{kg}$ CLZ following the dose in clinic.

Preparation of oral formulations containing CLZ. CLZ nanoparticles were prepared using the ball mill method.Zirconia balls (diameter: $10 \mathrm{~mm}$ ), DS, and MC were added to a zirconia cup (diameter: $45 \mathrm{~mm}$ ) containing CLZ or recrystallized CLZ (CLZ/R) and the mixture was crushed using a Pulverisette 7 for $24 \mathrm{~h}$ (400 rpm, room temperature, milled-CLZ). In addition, CLZ/R was prepared as follows: $0.5 \mathrm{~g}$ of CLZ was dissolved in $50 \mathrm{ml}$ of $50 \%$ ethanol at $120^{\circ} \mathrm{C}$, then the extracted CLZ was treated with sonication for $5 \mathrm{~s}$ using a sonicator (Yamato Science Co., Ltd, Tokyo, Japan) and allowed to stand for $24 \mathrm{~h}$. Thereafter, CLZ/R was collected by filtration (recovery rate: 92.3\%). These micro- and nanoparticles of CLZ and CLZ/R were dispersed using 5\% HP $\beta C D$ solution containing gum arabic (solubility at room temperature is approximately $500 \mathrm{mg} / \mathrm{ml}$ in water), polyvinylpyrrolidone (PVP, solubility at room temperature is approximately $>100 \mathrm{mg} / \mathrm{ml}$ in water), and D-mannitol (mannitol, solubility at room temperature is approximately $200 \mathrm{mg} / \mathrm{ml}$ in water) and the mixture was freeze-dried for 3 days (CLZ tablet, major axis $11.91 \pm 0.02 \mathrm{~mm}$, minor axis $7.05 \pm 0.01 \mathrm{~mm}$, thickness $6.87 \pm 0.02 \mathrm{~mm}$, weight $153.0 \pm 2.5 \mathrm{mg}$, CLZ content $22.3 \pm 0.7 \mathrm{mg} /$ tablet, disintegration time $30 \pm 6 \mathrm{sec}$, mean \pm standard error, $n=10$ ). The oral CLZ formulations (tablets) are presented in Table I. The CLZ tablets were suspended in purified water (re-dispersion of CLZ tablet) and used for the in vitro, in situ, and in vivo studies. The particle size was measured using a nanoparticle size analyzer (SALD-7100, Shimadzu Corp., Kyoto, Japan; refractive index 1.60-0.10i).

Characterization of CLZ. The morphology of CLZ was characterized using a scanning electron microscope (SEM) and powder X-ray diffraction (XRD). The SEM (JSM-5200, JEOL, Japan) was operated at an excitation voltage of $20 \mathrm{kV}$. The samples were fixed on a brass stub using carbon double-sided tape and deposited and gold-plated prior to recording images. After the degree of the vacuum reached 0.2-0.02 Torr, the gold plating was performed for $100-120 \mathrm{sec}$ to form a $10-20 \mathrm{~nm}$ gold layer. The XRD patterns of the samples were recorded using a SmartLab $2 \mathrm{~kW}$ (Rigaku, Co., Tokyo, Japan) with a $\mathrm{Cu}-\mathrm{Ka}(\lambda=1.54 \AA)$ target. The $\mathrm{X}$-rays were done at $40 \mathrm{kV}$ and $40 \mathrm{~mA}$ Philips. Data were obtained from $5^{\circ}$ to $40^{\circ}$ diffraction angles with a scanning rate of $3 \% \mathrm{~min}$ and a step size of 0.02 .

In situ intestinal absorption of CLZ. The experiment was performed according to our previous reports (22). Wistar rats were fasted for $10 \mathrm{~h}$ before dosing and allowed free access to water throughout the experiment. Rats were anesthetized with isoflurane and placed on a heating mat to maintain a body temperature of approximately $37^{\circ} \mathrm{C}$. A target portion of intestine $(4-5 \mathrm{~cm})$ was exposed through a midline abdominal incision and silicon tubing (TERUMO Corp., Tokyo, Japan) was inserted in one end of the intestine. The opposite end was tied and 1.5-2.0 ml of $3 \mathrm{mg} / \mathrm{kg}$ CLZ suspension (CLZ tablet in purified water) was injected through the tube. Heparin $(10 \mathrm{mg} / \mathrm{kg})$ was injected into the femoral vein. The mesenteric vein was cannulated with an appropriate size of polyethylene tubing (Hibiki Co., Tokyo, Japan) and blood samples (0.1 ml) were collected from a portal vein at 0 (pre-dose), $5,10,15,20$, 25 , and $30 \mathrm{~min}$ after dosing. Plasma samples were obtained by centrifugation $\left(20,400 \mathrm{x} \mathrm{g}, 20 \mathrm{~min}, 4^{\circ} \mathrm{C}\right)$. The amount of CLZ in the filtrates was determined using a Shimadzu LC-10AD system equipped with a CTO-6A column oven (Shimadzu Corp., Kyoto, Japan; HPLC method). The conditions were as follows: Internal standard, benzophenone; column, Inertsil ODS-3 ( $3 \mu \mathrm{m}$, column size: 2.0x50 mm; Shimadzu Co., Inc., Tokyo, Japan); mobile phase, acetonitrile/methanol/water $(35 / 15 / 50, \mathrm{v} / \mathrm{v} / \mathrm{v})$; flow rate, $0.25 \mathrm{ml} / \mathrm{min}$; column temperature, $35^{\circ} \mathrm{C}$; wavelength, $254 \mathrm{~nm}$.

Analysis of CLZ concentration in the stomach and small intestine after oral administration. The experiment was performed according to our previous studies (17). CLZ tablets were suspended using purified water and orally administered to Wistar rats at a dose of $3 \mathrm{mg} / \mathrm{kg}$. The rats were fasted for $10 \mathrm{~h}$ before oral administration of CLZ, but had free access to water. The rats were killed under deep ether anesthesia 3, 6, and $9 \mathrm{~h}$ later. The stomach and small intestine (40\% of the upper part of total small intestine, approximately $28 \mathrm{~cm}$ ) were excised and these mucous membrane samples $(0.1 \mathrm{mg})$ were homogenized in $300 \mu \mathrm{l}$ of methanol and centrifuged $\left(20,400 \mathrm{x} \mathrm{g}, 20 \mathrm{~min}, 4^{\circ} \mathrm{C}\right)$. The amount of CLZ in the supernatant was analyzed using the HPLC method described above.

Analysis of plasma CLZ concentration. CLZ tablets were suspended using purified water and orally administered to Wistar rats at a dose of $3 \mathrm{mg} / \mathrm{kg}$. The rats were fasted for $10 \mathrm{~h}$ before dosing and allowed free access to water throughout the experiment. Blood samples $(0.1 \mathrm{ml})$ were collected from a jugular vein at 0 (pre-dose), $0.5,1,2,4,6$, and $8 \mathrm{~h}$ after oral administration of CLZ and centrifuged (20,400 x g, $20 \mathrm{~min}$, $4^{\circ} \mathrm{C}$ ). The amount of CLZ in the supernatant was determined using the HPLC method described above. 
Table I. Formulations of CLZ tablets.

\begin{tabular}{lcccccccc}
\hline & \multicolumn{8}{c}{ Content (w/w\%) } \\
\cline { 2 - 6 } Formulation & CLZ & DS & MC & HPßCD & Gum arabic & PVP & Mannitol n & Treatment \\
\hline CLZ $_{\text {micro }}$ tablet & 17 & 4 & 17 & 2.5 & 47 & 2.5 & 10 & - \\
CLZ/R $_{\text {micro }}$ tablet & 17 & 4 & 17 & 2.5 & 47 & 2.5 & 10 & Recrystallization \\
CLZ $_{\text {nano }}$ tablet & 17 & 4 & 17 & 2.5 & 47 & 2.5 & 10 & Ball mill \\
CLZ/R $_{\text {nano }}$ tablet & 17 & 4 & 17 & 2.5 & 47 & 2.5 & 10 & Recrystallization, Ball mill \\
\hline
\end{tabular}

CLZ, cilostazol; DS, docusate sodium; HP $\beta C D$, 2-hydoxypropyl- $\beta$-cyclodextrin; PVP, polyvinylpyrrolidone.

The CLZ concentration in the plasma after a single injection of $0.3 \mathrm{ml}$ of CLZ solution $(100 \mu \mathrm{g} / \mathrm{kg})$ into the femoral vein was analyzed according to equation 1 :

$$
C_{\mathrm{CLZ}}=C_{0} \cdot e^{-k_{\mathrm{e}} \cdot t}
$$

where $C_{\text {CLZ }}$ was the plasma CLZ concentration, $C_{0}$ was the initial concentration of CLZ in the plasma $(4.10 \pm 0.13 \mu \mathrm{g} / \mathrm{ml}), k_{\mathrm{e}}$ was the elimination rate constant for CLZ from the plasma $\left(2.05 \pm 0.31 \mathrm{~h}^{-1}\right)$, and $V_{\mathrm{d}}$ was the distribution volume $(30.5 \pm 1.52 \mu \mathrm{l} / \mathrm{kg})$. These data were obtained from three experiments.

The absorption of CLZ after the single administration of a CLZ tablet was calculated as the apparent absorption rate constant $\left(k_{\mathrm{a}}, \mathrm{h}^{-1}\right)$ according to equation 2 :

$$
C_{\mathrm{CLZ}}=\frac{k_{\mathrm{a}} \cdot F \cdot D}{V_{\mathrm{d}}\left(k_{\mathrm{a}}-k_{\mathrm{e}}\right)}\left(e^{-k_{\mathrm{e}} \cdot\left(t t_{\mathrm{bg}}\right)}+e^{-k_{\mathrm{a}} \cdot\left(t-t_{\mathrm{Lg}}\right)}\right)
$$

where $k_{\mathrm{a}}$ was the absorption rate constant, $t$ was the time after CLZ administration ( $0-8 \mathrm{~h}$ ), $F$ was the fraction of CLZ absorbed, $D$ was the dose of CLZ administered $(3 \mathrm{mg} / \mathrm{kg})$, and $t_{\text {lag }}$ was the lag time (h). The area under the CLZ concentration-time curve (AUC) was determined according to the trapezoidal rule up to $8 \mathrm{~h}$. The mean residence time (MRT) was calculated from the area under the first moment curve (AUMC)/AUC. In addition, the bioavailability was calculated as the ratio of the AUC after oral administration to the AUC after intravenous administration.

Induction of focal cerebral ischemia/reperfusion model. Focal cerebral ischemia/reperfusion was caused by middle cerebral artery occlusion (MCAO), which was completed following the method described in our previous reports (15). MCAO was induced by the insertion of a silicone-coated 8-0 nylon monofilament into the left middle cerebral artery under isoflurane anesthesia. Two $h$ after this procedure, the mice were briefly reanesthetized with isoflurane and middle cerebral artery blood flow was restored by withdrawing the nylon monofilament. Three $\mathrm{h}$ after reperfusion, CLZ suspension (CLZ tablet in purified water) was orally administered to the MCAO mice at a dose of $3 \mathrm{mg} / \mathrm{kg}$. Three days after reperfusion, the brain was removed and three slices were removed for analysis: i) from the bregma ( $2 \mathrm{~mm}$, Area $\left._{\mathrm{A}}\right)$, ii) $1 \mathrm{~mm}$ anterior to the bregma (2 $\mathrm{mm}$, Area $\left.\mathrm{B}_{\mathrm{B}}\right)$, and iii) $1 \mathrm{~mm}$ posterior to the bregma ( $2 \mathrm{~mm}$, Area $\mathrm{C}_{\mathrm{C}}$, using Brain Matrices (Brain ScienceIdea Co., Ltd., Osaka, Japan). The slices of brain tissue were dyed with 1.5\% 2,3,5-triphenyl tetrazolium chloride (TTC) for $15 \mathrm{~min}$ and monitored under a digital camera. The images obtained were analyzed using Image $\mathbf{J}$ software and the infarct area was calculated $\left(\mathrm{mm}^{2}\right)$. The infarct volume $\left(\mathrm{mm}^{3}\right)$ was estimated according to the following equation 3 :

$$
\text { Infarct volume }\left(\mathrm{mm}^{3}\right)=\operatorname{Area}_{\mathrm{A}} \times 2+\operatorname{Area}_{\mathrm{B}} \times 2+\operatorname{Area}_{\mathrm{C}} \times 2
$$

Neurological deficit mice were tested for neurological deficits $72 \mathrm{~h}$ after MCAO followed by reperfusion (MCAO/reperfusion). Each mouse was masked for the investigator.

Statistical Analysis. Unpaired Student's $t$-tests and Dunnett's multiple comparison tests were used for statistical analysis and P-values less than 0.05 were considered significant. All data are expressed as the mean \pm standard deviation or standard error of the mean.

\section{Results}

Preparation of tablets containing CLZ nanoparticles. Fig. 1 shows the XRD patterns (A, B) and SEM images (C, D) of CLZ and CLZ/R. The transparent acicular crystal appeared after recrystallization. In the XRD patterns, the indicated CLZ peak forms of polymorphs were observed and CLZ consisted of Forms A, B, and C (Fig. 1A). However, the indicated peak of Form $\mathrm{C}$ decreased and the indicated peak of Form A was enhanced (Fig. 1B). Thereafter, we prepared CLZ tablets containing CLZ micro- and nanoparticles using the ball mill method and evaluated them for re-dispersibility. The mean particle sizes of CLZ, CLZ/R, milled-CLZ, and milled-CLZ/R were $65.4 \pm 26.7,48.3 \pm 34.4,0.067 \pm 0.018$, and $0.073 \pm 0.016 \mu \mathrm{m}$, respectively (means \pm standard deviation). The tablets containing CLZ as described in Table I were prepared using CLZ and CLZ/R with or without the ball mill. Fig. 2 shows the particle size distribution of the $\mathrm{CLZ}_{\text {micro }}(\mathrm{A}), \mathrm{CLZ} / \mathrm{R}_{\text {micro }}$ (B), $\mathrm{CLZ}_{\text {nano }}(\mathrm{C})$, and CLZ/R $\mathrm{R}_{\text {nano }}$ (D) tablets. Even though the particles in $\mathrm{CLZ}_{\text {nano }}$ tablets $(2.79 \pm 82.1 \mu \mathrm{m})$ aggregated after re-dispersion, the mean particle size of the CLZ/ $\mathrm{R}_{\text {nano }}$ tablets $(0.068 \pm 0.029 \mu \mathrm{m})$ was similar to that of the milled-CLZ/R $\mathrm{R}_{\text {nano }}$ tablets (means \pm standard deviation). 

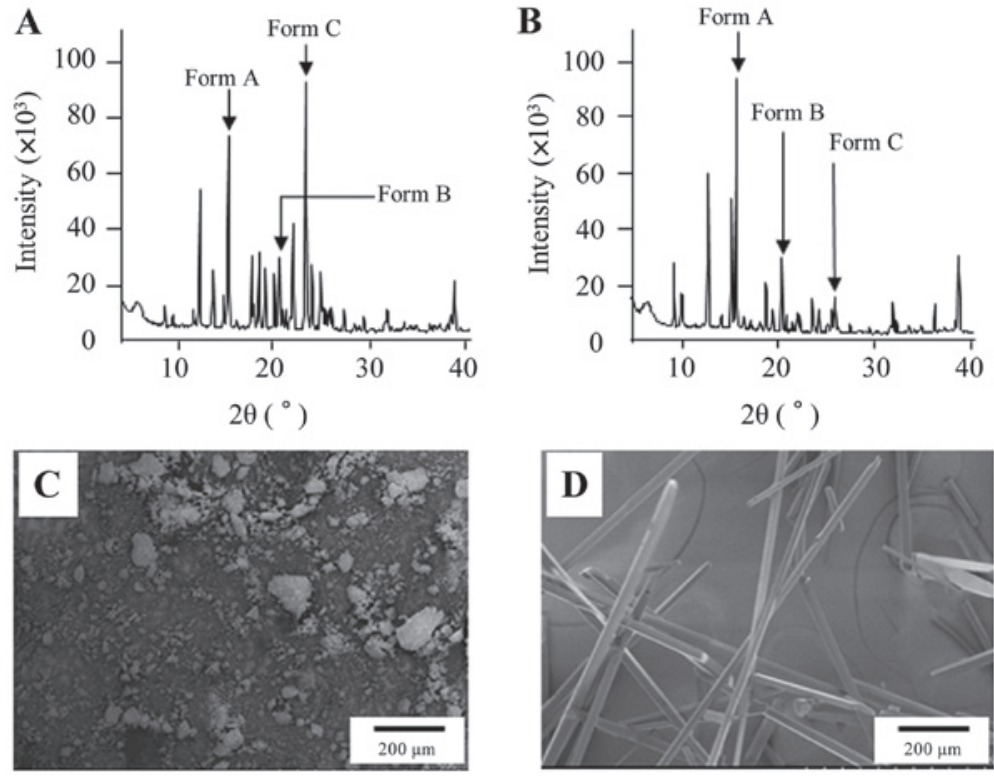

Figure 1. XRD patterns and SEM images of CLZ with or without recrystallization. (A and B) XRD pattern of (A) CLZ and (B) CLZ/R. (C and D) SEM image of (C) CLZ and (D) CLZ/R. Arrows show the indicated peak of CLZ forms of polymorphs (Form A, $2 \theta=13.0 \pm 0.2 ;$ Form B, 2 $\theta=21.5 \pm 0.2^{\circ}$; Form C, 25.0 \pm 0.2 ). XRD, X-ray diffraction; SEM, scanning electron microscope; CLZ, cilostazol.
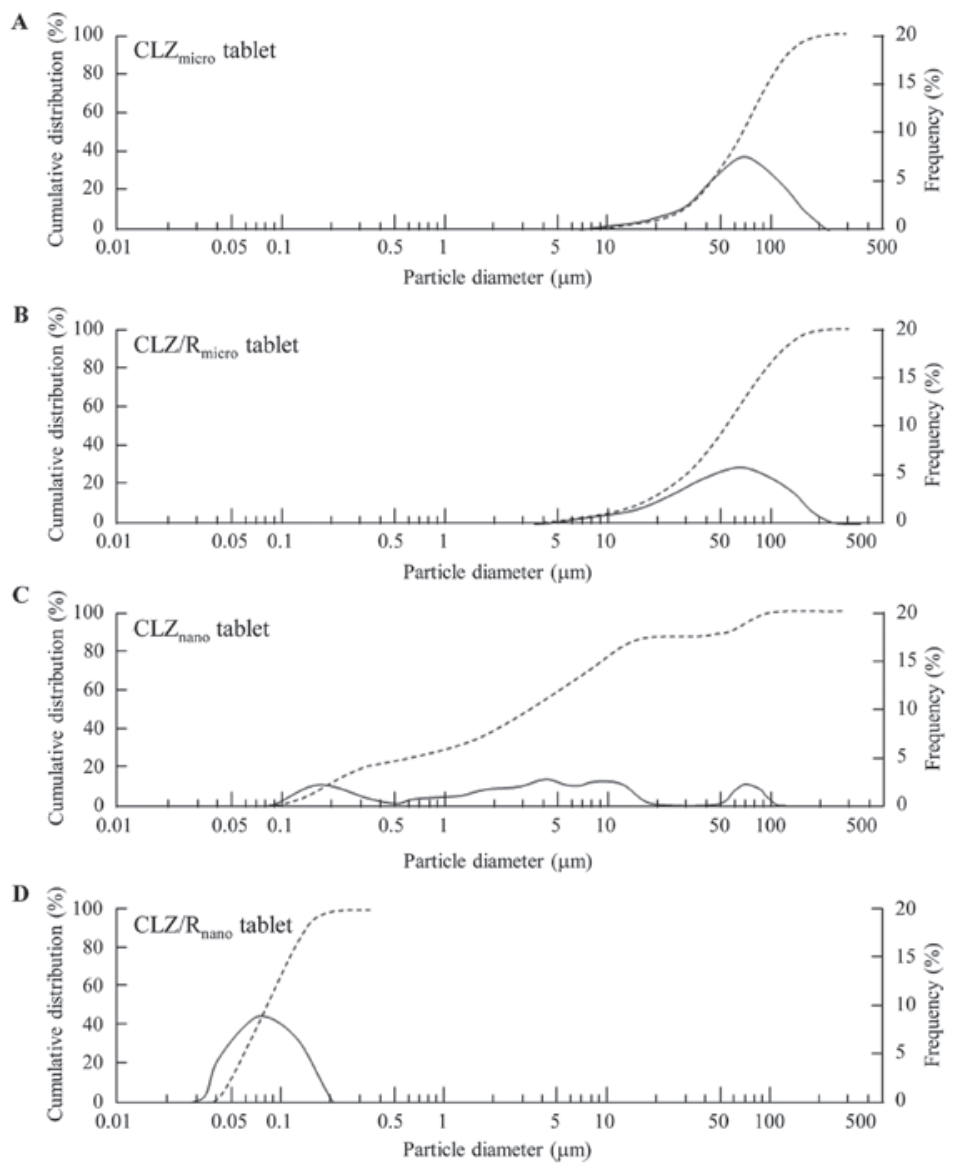

Figure 2. Cumulative size distribution (dashed line) and frequency (solid line) of CLZ after tablet re-dispersion. The composition of the tablets containing CLZ

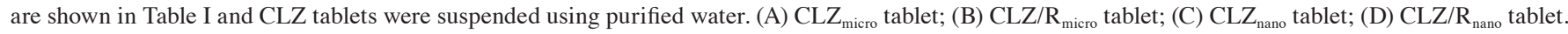
The mean particle size after re-dispersion of $C L Z_{\text {micro }}, C L Z / R_{\text {micro }}, C L Z_{\text {nano }}$, and CLZ/ $R_{\text {nano }}$ tables was $63.2 \pm 0.267,48.3 \pm 0.312,2.79 \pm 0.821$, and $0.064 \pm 0.047 \mu \mathrm{m}$, respectively (means \pm standrad deviation). CLZ, cilostazol.

Pharmacokinetics of the oral formulation containing CLZ nanoparticles. The in situ loop technique was used to evaluate the drug absorption of the CLZ/R tablet and Fig. 3 shows the difference in drug absorption between the CLZ/ $\mathrm{R}_{\text {micro }}$ and 


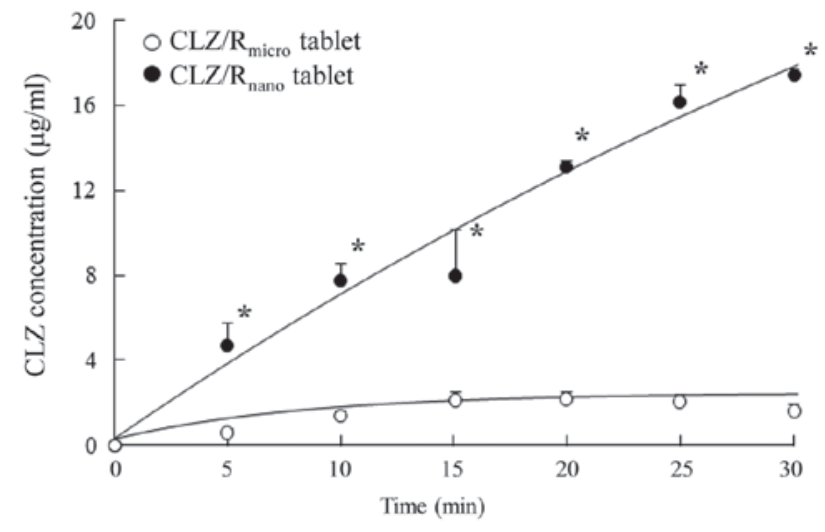

Figure 3. Changes in plasma CLZ concentration after administration of CLZ/R tablet into the small intestine. The composition of the tablets containing CLZ are shown in Table I. The CLZ tablets were suspended using purified water and administered to rats $(3 \mathrm{mg} / \mathrm{kg})$. Open circles (CLZ/R $\mathrm{R}_{\text {micro }}$ tablet), CLZ/ $\mathrm{R}_{\text {micro }}$ tablet-treated rats; closed circles (CLZ $/ \mathrm{R}_{\text {nano }}$ tablet), CLZ $/ \mathrm{R}_{\text {nano }}$ tablet-treated rats. Means \pm standard error, $n=3-5$. ${ }^{*} \mathrm{P}<0.05$, vs. CLZ/ $\mathrm{R}_{\text {micro }}$ tablet groups for each category. CLZ, cilostazol.

$\mathrm{CLZ} / \mathrm{R}_{\text {nano }}$ tablets. The absorption of CLZ/ $\mathrm{R}_{\text {nano }}$ tablets was significantly higher than that of CLZ/R ${ }_{\text {micro }}$ tablets. Fig. 4 shows the amounts of CLZ in the mucosal membrane of the rat stomach and small intestine after oral administration of $\mathrm{CLZ} / \mathrm{R}_{\text {micro }}$ and $\mathrm{CLZ} / \mathrm{R}_{\text {nano }}$ tablets. The residence amount and time after CLZ/R $\mathrm{R}_{\text {nano }}$ tablet administration were significantly greater than those after $\mathrm{CLZ} / \mathrm{R}_{\text {micro }}$ tablet administration. The amount of CLZ in mucosal membrane of the stomach reached a maximum $3 \mathrm{~h}$ after administration of $\mathrm{CLZ} / \mathrm{R}_{\text {micro }}$ and $\mathrm{CLZ} / \mathrm{R}_{\text {nano }}$ tablets, then decreased with time. After administration of the CLZ ${ }_{\text {micro }}$ tablet, the amount of CLZ in the mucosal membrane of the small intestine also reached a maximum after $3 \mathrm{~h}$. However, the amount of CLZ in the mucosal membrane of the small intestine remained constant during the period 3-12 h after administration of CLZ/R $\mathrm{R}_{\text {nano }}$ tablets. Fig. 5 shows the plasma CLZ concentrations after oral administration of $\mathrm{CLZ} / \mathrm{R}_{\text {micro }}$ and $\mathrm{CLZ} / \mathrm{R}_{\text {nano }}$ tablets in rats and Table II summarizes the pharmacokinetic parameters calculated from the in vivo intestinal penetration data. The plasma CLZ levels, AUC, and MRT in rats administered CLZ/ $R_{\text {nano }}$ tablets were all significantly higher than those in rats administered CLZ/ $\mathrm{R}_{\text {micro }}$ tablets. Moreover, the bioavailability of the $\mathrm{CLZ} / \mathrm{R}_{\text {nano }}$ tablets was 2.1 -fold higher than that of the $\mathrm{CLZ} / \mathrm{R}_{\text {micro }}$ tablets.

Therapeutic effect of the oral formulation containing CLZ/R nanoparticles on ischemic stroke. Fig. 6 shows the effects on ischemic stroke in $\mathrm{MCAO} /$ reperfusion mice administered $\mathrm{CLZ} / \mathrm{R}_{\text {nano }}$ tablets. Treatment with $\mathrm{CLZ} / \mathrm{R}_{\text {nano }}$ tablets attenuated ischemic stroke and the infarct volume of the MCAO/reperfusion mice administered CLZ/ $\mathrm{R}_{\text {nano }}$ tablets was $31 \%$ of that of the vehicle-administered mice (vehicle). The neurological deficits in $\mathrm{MCAO} /$ reperfusion mice were also protected after the oral administration of $\mathrm{CLZ} / \mathrm{R}_{\text {micro }}$ and CLZ/ $\mathrm{R}_{\text {nano }}$ tablets (vehicle, 2.7 $\pm 0.3 ; \mathrm{CLZ} / \mathrm{R}_{\text {nano }}$ tablet, $1.2 \pm 0.3$, means \pm standard error, $\mathrm{n}=3-9$ ).

\section{Discussion}

Poorly water-soluble drugs are poorly absorbed when administered orally. We previously designed drug nanoparticles by
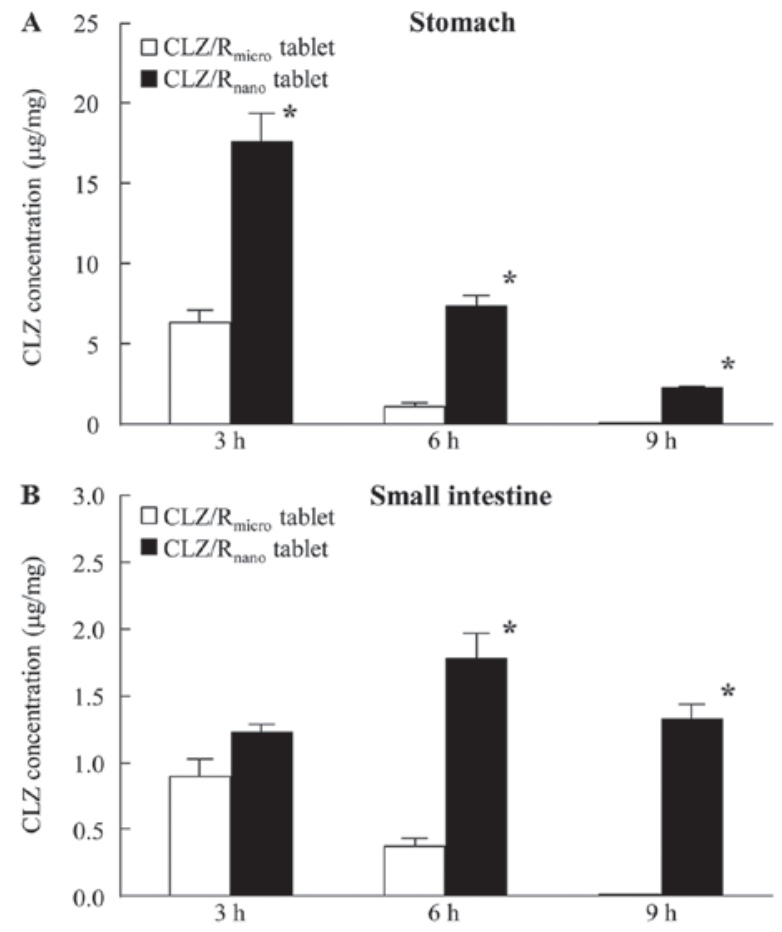

Figure 4. Changes in the amount of CLZ in the mucosal membrane of the (A) stomach and (B) small intestine after oral administration of CLZ/R tablets. The composition of the tablets containing CLZ are shown in Table I and the CLZ tablets were suspended using purified water. The rats were fasted for $18 \mathrm{~h}$ before the experiments, but had free access to water. The rats were orally administered CLZ/ $\mathrm{R}_{\text {micro }}$ or CLZ/ $\mathrm{R}_{\text {nano }}$ tablets $(3 \mathrm{mg} / \mathrm{kg})$ and killed under deep ether anesthesia 3,6 , or $9 \mathrm{~h}$ later. Open columns $\left(\mathrm{CLZ} / \mathrm{R}_{\text {micro }}\right.$ tablet), CLZ/ $\mathrm{R}_{\text {micro }}$ tablet-administered rats; closed columns $\left(\mathrm{CLZ} / \mathrm{R}_{\text {nano }}\right.$ tablet), $C L Z / R_{\text {nano }}$ tablet-administered rats. Means \pm standard error, $n=3-5$. ${ }^{*} \mathrm{P}<0.05$, vs. CLZ/R $\mathrm{R}_{\text {micro }}$ tablet groups for each category. CLZ, cilostazol.

the breakdown method using a ball and bead mill (13-18) and showed that indomethacin solid nanoparticles enhanced drug bioavailability in the small intestine of rats (17). In this study, we designed new oral formulations containing CLZ solid nanoparticles using a combination of recrystallization and breakdown methods. Moreover, we investigated the therapeutic effect of the oral formulations containing CLZ nanoparticles on ischemic stroke in MCAO/reperfusion mice.

First, we attempted to prepare an oral formulation (tablet) containing CLZ nanoparticles. Stowell et al (23)and Whittall et al (24) reported that three forms of CLZ polymorphs exist (Form A, B and C). Forms A, B, and C were characterized by XRD and had the indicated peaks at $2 \theta=13.0 \pm 0.2$ (Form A), $2 \theta=21.5 \pm 0.2$ (Form B), and $2 \theta=25.0 \pm 0.2^{\circ}$ (Form C). In this study, CLZ that was not recrystallized (original CLZ powder) consisted of a mix of Forms A, B and C. However, the peak of Form B was decreased after recrystallization and that of Form A was decreased in recrystallized CLZ (CLZ/R) (Fig. 1). Furthermore, we prepared an oral formulation containing CLZ nanoparticles using the ball mill method and evaluated the changes in CLZ particle size after the re-dispersion of the tablets (Fig. 2). The particles aggregated after the $\mathrm{CLZ}_{\text {nano }}$ tablets were re-dispersed; however, the stability of the re-dispersed oral formulation was improved when recrystallized CLZ was used. In addition, the particle size after re-dispersion of the $C L Z / R_{\text {nano }}$ tablets was similar to that of CLZ/R prepared with the ball mill. The stability of 
Table II. Pharmacokinetic parameters for plasma CLZ concentration after oral administration of CLZ/R tablet.

\begin{tabular}{lcccc}
\hline Preparation & $\mathrm{k}_{\mathrm{a}}(/ \mathrm{h})$ & $\mathrm{t}_{\text {lag }}(\mathrm{h}) \times 10^{-2}$ & Fx 10 & AUC $(\mu \mathrm{g} \bullet \mathrm{h} / \mathrm{ml})$ \\
\hline $\mathrm{CLZ} / \mathrm{R}_{\text {micro }}$ tablet & $0.31 \pm 0.07$ & $7.61 \pm 4.12$ & $1.41 \pm 0.19$ & $0.94 \pm 0.11$ \\
$\mathrm{CLZ} / \mathrm{R}_{\text {nano }}$ tablet & $0.25 \pm 0.03$ & $6.04 \pm 2.70$ & $2.81 \pm 0.26^{\mathrm{a}}$ & $1.71 \pm 0.08^{\mathrm{a}}$ \\
\hline
\end{tabular}

Parameters were calculated according to equations 1 and 2 (see Materials and methods). The compositions of the CLZ tablet are shown in Table I. The data are presented as means \pm standard error of 3 independent rats. ${ }^{a} \mathrm{P}<0.05$, vs. CLZ/ $\mathrm{R}_{\text {micro }}$ tablet for each category. CLZ, cilostazol; AUC, concentration-time curve; MRT, mean residence time.

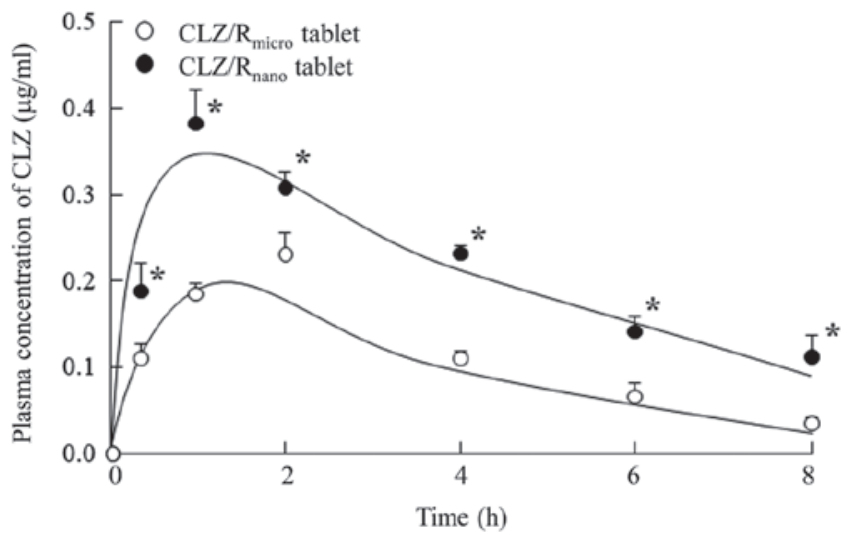

Figure 5. Plasma CLZ concentrations following oral administration of CLZ/R tablets. The composition of the tablets containing CLZ are shown in Table I and the CLZ tablets were suspended using purified water. The rats were fasted for $18 \mathrm{~h}$ before the experiments, but had free access to water. The rats were orally administered CLZ/ $\mathrm{R}_{\text {micro }}$ or CLZ/R $\mathrm{R}_{\text {nano }}$ tablets $(3 \mathrm{mg} / \mathrm{kg})$. Solid lines present fitting curves calculated using equation 2 (see Materials and methods). Open columns (CLZ/R $\mathrm{R}_{\text {micro }}$ tablet), CLZ/R $\mathrm{R}_{\text {micro }}$ tablet-administered rats; closed columns $\left(\mathrm{CLZ} / \mathrm{R}_{\text {nano }}\right.$ tablet $), \mathrm{CLZ} / \mathrm{R}_{\text {nano }}$ tablet-administered rats. Means \pm standard error, $n=3$. ${ }^{*} \mathrm{P}<0.05$, vs. $C L Z / \mathrm{R}_{\text {micro }}$ tablet groups for each category. CLZ, cilostazol.

the nanoparticles was improved by changing the ratio of the forms of polymorphs in the recrystallized CLZ. The relationship between the polymorph forms and their stability after re-dispersion will be examined in a future study.

Thereafter, we evaluated drug absorption and residence time in the small intestine after the oral administration of $\mathrm{CLZ} / \mathrm{R}_{\text {micro }}$ and CLZ/ $\mathrm{R}_{\text {nano }}$ tablets. The absorption, residence amount, and residence time of the $C L Z / R_{\text {nano }}$ tablets were significantly greater than those of the $C L Z / R_{\text {micro }}$ tablets (Figs. 3-5). Furthermore, we investigated the pharmacokinetics after the oral administration of $\mathrm{CLZ} / \mathrm{R}_{\text {micro }}$ and $\mathrm{CLZ} / \mathrm{R}_{\text {nano }}$ tablets. The plasma CLZ levels, AUC, and MRT in rats administered $C L Z / R_{\text {nano }}$ tablets were also increased compared with those in rats administered CLZ/ $\mathrm{R}_{\text {micro }}$ tablets (Table II and Fig. 5). In addition, the absorption of $C L Z / R_{\text {nano }}$ tablets was higher than that of the commercially available CLZ OD tablets (AUC, $0.55 \pm 0.05$, means \pm standard error, $n=5$ ). These results indicated that bioavailability was enhanced after administration of CLZ solid nanoparticles.

It is important to clarify the mechanism of the enhancement of bioavailability that is exhibited by the administration of nanoparticles. The residue of CLZ in the stomach and small intestine after oral administration of the CLZ/ $\mathrm{R}_{\text {nano }}$ tablets was significantly increased compared to that of the $C L Z / R_{\text {micro }}$
A
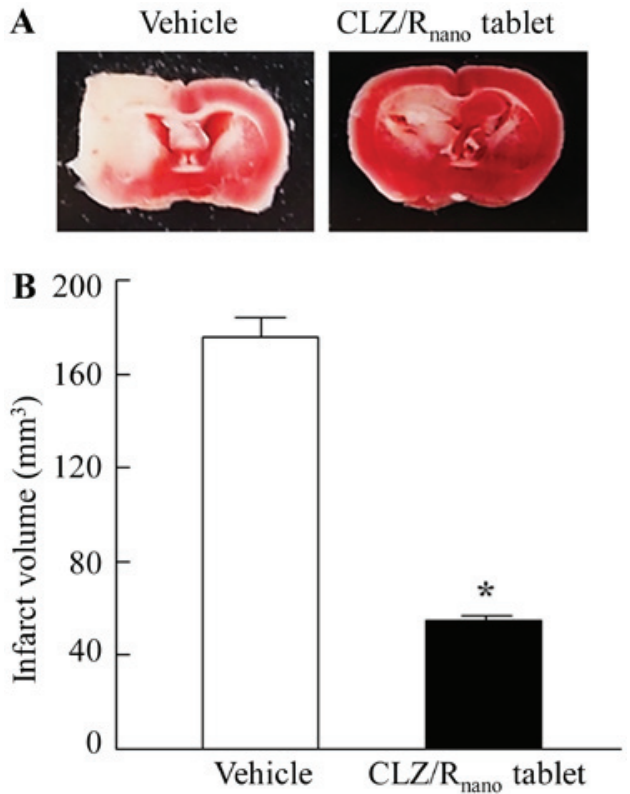

Figure 6. Effect of CLZ/R tablets on ischemic stroke in MCAO/reperfusion mice. Image of the (A) brain and (B) infarct volume in $\mathrm{MCAO} /$ reperfusion mice administered CLZ/R tablets $(3 \mathrm{mg} / \mathrm{kg}$ ). Infarct volumes were calculated according to equation 3 (see Materials and methods). Vehicle, vehicle-administered mice; $C L Z / R_{\text {nano }}$ tablet, CLZ/ $R_{\text {nano }}$ tablet-administered mice. Means \pm standard error, $n=3-9$. $P<0.05$, vs. vehicle groups for each category. CLZ, cilostazol.

tablets (Fig. 4). In addition, we previously reported that the nanoparticle formulation of indomethacin enhanced the permeability of the corneal and intestinal membrane compared with the microparticle formulation $(17,18)$. Taking these findings together, we hypothesized that the character of the solid nanoparticles caused the enhancement of the bioavailability of $\mathrm{CLZ} / \mathrm{R}_{\text {nano }}$ tablets.

CLZ is widely used for the secondary prevention of cerebral infarction. Recently, Kasahara et al (25) reported that treatment with CLZ suppressed the disruption of the microvasculature in ischemic areas. In addition, we reported that the intravenous administration of CLZ attenuated brain damage when administered immediately after the onset of ischemic stroke symptoms in MCAO/reperfusion mice (15). From these findings, we investigated whether the oral administration of CLZ/R tablets prevented ischemic stroke in MCAO/reperfusion mice and found that $C L Z / R_{\text {nano }}$ tablets attenuated ischemic stroke (Fig. 6). This result indicated that CLZ/R, in which the crystal structure was changed, also had a protective effect on ischemic stroke. Further studies are needed to 
elucidate the usefulness of the oral formulation containing CLZ nanoparticles in the treatment of ischemic stroke. In addition, it is important to clarify the relationships between crystal structure and stability after the re-dispersion of CLZ tablets. Therefore, we are now investigating the effect of each of the CLZ polymorphic forms (Form A-C) in the CLZ/R tablet on stability in re-dispersion, drug absorption, and treatment of ischemic stroke. In addition, we demonstrated the difference in the preventive effects on ischemic stroke between CLZ micro and nanoparticles with or without recrystallization using MCAO/reperfusion mice.

In conclusion, we prepared a novel oral formulation containing CLZ nanoparticles (CLZ/ $\mathrm{R}_{\text {nano }}$ tablet) that exhibited a high stability after re-dispersion and a high drug bioavailability. In addition, the CLZ/ $\mathrm{R}_{\text {nano }}$ tablets ameliorated neurological deficits caused by ischemic stroke in MCAO/reperfusion mice. It is possible that the oral formulation containing CLZ/R nanoparticles may be useful for effectively treating ischemic stroke patients. These findings provide significant information that can be used to design further studies aimed at developing treatments for ischemic stroke patients and to improve drug bioavailability.

\section{References}

1. Kimura Y, Tani T, Kanbe T and Watanabe K: Effect of cilostazol on platelet aggregation and experimental thrombosis. Arzneimittelforschung 35: 1144-1149, 1985.

2. Kanbayashi J, Liu Y, Sun B, Shakur Y, Yoshitake M and Czerwiec F: Cilostazol as a unique antithrombotic agent. Curr Pharm Des 9: 2289-2302, 2003.

3. Bramer SL and Forbes WP: Relative bioavailability and effects of a high fat meal on single dose cilostazol pharmacokinetics. Clin Pharmacokinet 37 (Suppl 2): S13-S23, 1999.

4. Jinno J, Kamada N, Miyake M, Yamada K, Mukai T, Odomi M, Toguchi H, Liversidge GG, Higaki K and Kimura T: Effect of particle size reduction on dissolution and oral absorption of a poorly water-soluble drug, cilostazol, in beagle dogs. J Control Release 111: 56-64, 2006.

5. Jinno J, Kamada N, Miyake M, Yamada K, Mukai T, Odomi M, Toguchi H, Liversidge GG, Higaki $K$ and Kimura T: In vitro-in vivo correlation for wet-milled tablet of poorly water-soluble cilostazol. J Control Release 130: 29-37, 2008.

6. Lee JH, Park SY, Shin YW, Hong KW, Kim CD, Sung SM, Kim KY and Lee WS: Neuroprotection by CLZ, a phosphodiesterase type 3 inhibitor, against apoptotic white matter changes in rat after chronic cerebral hypoperfusion. Brain Res 1082: 182-191, 2006.

7. Rasenack N and Müller BW: Micron-size drug particles: Common and novel micronization techniques. Pharm Dev Technol 9: 1-13, 2004

8. Murakami H, Kobayashi M, Takeuchi H and Kawashima Y: Further application of a modified spontaneous emulsification solvent diffusion method to various types of PLGA and PLA polymers for preparation of nanoparticles. Powder Technol 107: $137-143,2000$
9. Kawashima Y: Design of poly (lactic-co-glycolic acid) (PLGA) nanosphere for developing to DDS. J Pharm Sci Technol Jpn 66: 224-238, 2006

10. Sha S, Vong LB, Chonpathompikunlert P, Yoshitomi T, Matsui H and Nagasaki Y: Suppression of NSAID-induced small intestinal inflammation by orally administered redox nanoparticles. Biomaterials 34: 8393-8400, 2013.

11. Igarashi R, Takenaga M, Takeuchi J, Kitagawa A, Matsumoto K and Mizushima Y: Marked hypotensive and blood flow-increasing effects of a new lipo-PGE(1) (lipo-AS013) due to vascular wall targeting. J Control Release 71: 157-164, 2001.

12. Kataoka K, Harada A and Nagasaki Y. Block copolymer micelles for drug delivery design, characterization and biological significance. Adv Drug Deliv Rev 47: 113-131, 2001.

13. Nagai N, Nakazawa Y, Ito Y, Kanai K, Okamoto $N$ and Shimomura Y: A nanoparticle-based ophthalmic formulation of dexamethasone enhances corneal permeability of the drug and prolongs its corneal residence time. Biol Pharm Bull 40: 1055-1062, 2017.

14. Nagai N, Ito Y, Okamoto N and Shimomura Y: Size effect of rebamipide ophthalmic nanodispersions on its therapeutic efficacy for corneal wound healing. Exp Eye Res 151: 47-53, 2016.

15. Nagai N, Yoshioka C, Ito Y, Funakami Y, Nishikawa $\mathrm{H}$ and Kawabata A: Intravenous administration of cilostazol nanoparticles ameliorates acute ischemic stroke in a cerebral ischemia/reperfusion-induced injury model. Int J Mol Sci 16: 29329-29344, 2015.

16. Nagai N, Yoshioka C, Tanabe W, Tanino T, Ito Y, Okamoto $\mathrm{N}$ and Shimomura Y: Effects of ophthalmic formulations containing cilostazol nanoparticles on retinal vasoconstriction in rats injected with endothelin-1. Pharm Anal Acta 6: 4, 2015.

17. Nagai $\mathrm{N}$ and Ito Y: Effect of solid nanoparticle of indomethacin on therapy for rheumatoid arthritis in adjuvant-induced arthritis rat. Biol Pharm Bull 37: 1109-1118, 2014.

18. Nagai N, Ito $\mathrm{Y}$, Okamoto $\mathrm{N}$ and Shimomura $\mathrm{Y}$ : A nanoparticle formulation reduces the corneal toxicity of indomethacin eye drops and enhances its corneal permeability. Toxicology 319: 53-62, 2014.

19. Choi M, Na K, Kim J, Sakamoto Y, Terasaki O and Ryoo R: Stable single-unit-cell nanosheets of zeolite MFI as active and long-lived catalysts. Nature 461: 246-249, 2009.

20. Mintova S, Olson NH, Valtchev V and Bein T: Mechanism of zeolite A nanocrystal growth from colloids at room temperature. Science 283: 958-960, 1999.

21. Tosheva L and Valtchev VP: Nanozeolites: Synthesis, crytallisation mechanism and applications. Chem Mater 17: 2494, 2005.

22. Nagai N and Ito Y: Delay of cataract development in the Shumiya cataract rat by water containing enhanced concentrations of magnesium and calcium. Curr Eye Res 32: 439-445, 2007.

23. Stowell GW, Behme RJ, Denton SM, Pfeiffer I, Sancilio FD, Whittall LB and Whittle RR: Thermally-prepared polymorphic forms of cilostazol. J Pharm Sci 91: 2481-2488, 2002.

24. Whittall LB, Whittle RR and Stowell GW: Polymorphic forms of cilostazol. Acta Cryst C 58: 0525-0527, 2002.

25. Kasahara Y, Nakagomi T, Matsuyama T, Stern D and Taguchi A: Cilostazol reduces the risk of hemorrhagic infarction after administration of tissue-type plasminogen activator in a murine stroke model. Stroke 43: 499-506, 2012. 